\section{Variation in sperm morphology variation among Afrotropical sunbirds}

4

Running head: Sperm morphology in sunbirds

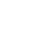

$$
\text { TAIWO C. OMOTORIOGUN, }{ }^{1,2 *} \text { TERJE LASKEMOEN, }{ }^{1} \text { MELISSAH ROWE, }{ }^{1,3} \text { TOMÁŠ }
$$$$
\text { ALBRECHT, }{ }^{4,5} \text { RAURI C. K. BOWIE, }{ }^{6} \text { ONDŘEJ SEDLÁČEK, }{ }^{7} \text { DAVID HOŘÁK, }{ }^{7} \text { ULF }
$$$$
\text { OTTOSSON }^{2} \& \text { JAN T. LIFJELD }{ }^{1}
$$

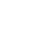

${ }^{1}$ Natural History Museum, University of Oslo, Oslo, Norway

${ }^{2}$ A.P. Leventis Ornithological Research Institute, University of Jos, Nigeria

${ }^{3}$ Centre for Ecological and Evolutionary Synthesis, Department of Biosciences, University of Oslo, Oslo, Norway

${ }^{4}$ Institute of Vertebrate Biology, Academy of Sciences of the Czech Republic, Brno, Czech Republic ${ }^{5}$ Department of Zoology, Faculty of Science, Charles University in Prague, Prague, Czech Republic ${ }^{6}$ Department of Integrative Biology and Museum of Vertebrate Zoology, University of California Berkeley, USA

${ }^{7}$ Department of Ecology, Faculty of Science, Charles University in Prague, Prague, Czech Republic

\section{${ }^{*}$ Corresponding author.}

Email: t.c.omotoriogun@nhm.uio.no 
Birds show considerable variation in sperm morphology. Closely related species and subspecies can show diagnostic differences in sperm size. There is also variation in sperm size among males within a population, and recent evidence from passerine birds suggests that the coefficient of inter-male variation in sperm length is negatively associated with the level of sperm competition. Here we examined patterns of inter- and intra-specific variation in sperm length in 12 species of sunbird (Nectariniidae) from Nigeria and Cameroon, a group for which such information is extremely limited. We found significant variation among species in sperm total length, with mean values ranging from 74 $\mu \mathrm{m}$ to $116 \mu \mathrm{m}$, placing these species within the short to medium sperm length range for passerine birds. Most of this variation was explained by the length of the midpiece, which contains the fused mitochondria and is an important structure for sperm energetics. Relative midpiece length was negatively correlated with the coefficient of inter-male variation in sperm total length across species, suggesting that sperm competition may have selected for greater midpiece length in this group. We also mapped sperm lengths onto a time-calibrated phylogeny and found support for a phylogenetic signal in all sperm length components, except head length. A test of various evolutionary or tree transformation models gave strongest support for the Brownian motion model for all sperm components, i.e. divergences were best predicted by the phylogenetic distance between lineages. The coefficients of inter-male variation in sperm total length indicate that sperm competition is high, but variable among sunbird species, similar to passerine birds at large.

Key words: comparative analysis, phylogenetic signal, sperm size, sperm competition, Nectariniidae 
Across the animal kingdom, spermatozoa vary remarkably in size, shape and behaviour (Cohen 1977, Pitnick et al. 2009, Pizzari \& Parker 2009). In passerine birds, sperm length varies from approximately $40 \mu \mathrm{m}$ to nearly $300 \mu \mathrm{m}$ (Pitnick et al. 2009, Lifjeld et al. 2010, Immler et al. 2011). Given that the primary role of sperm is to fertilize ova, a highly conserved function, the evolutionary diversification of sperm form is surprising and the factors generating this diversity are poorly understood (Snook 2005, Pitnick et al. 2009). However, it is generally thought that genetic drift, mode of fertilization and postcopulatory sexual selection, i.e. sperm competition (Parker 1970) and cryptic female choice (Eberhard 1996), drive evolutionary changes in sperm phenotypes (Franzén 1970, Snook 2005, Pitnick et al. 2009).

There is comparative evidence from a range of taxonomic groups that sperm length tends to increase with sperm competition, for examples in birds (Briskie et al. 1997, Kleven et al. 2009), insects (Morrow \& Gage 2000), fish (Balshine et al. 2001) and mammals (Gomendio \& Roldan 1991, Tourmente et al. 2011), although with some exceptions to this pattern (e.g. Gage \& Freckleton 2003, Immler \& Birkhead 2007). It is suggested that the evolution of longer sperm is driven by their ability to swim faster (Gomendio \& Roldan 1991), live longer (Parker 1993, Parker 1998) or displace shorter sperm from female sperm storage sites (Miller \& Pitnick 2002, Lüpold et al. 2012). In passerine birds, increased sperm size is associated with a disproportionate increase in the size of the midpiece (Lüpold et al. 2009), which contains a single fused mitochondrion wrapped helically around the flagellum (Koehler 1995). A longer midpiece contains more adenosine triphosphate (Rowe et al. 2013), thus demonstrating the importance of this structure for sperm energetics. Sperm length in passerine birds is also positively correlated with the length of the sperm storage tubules in females (Briskie \& Montgomerie 1992, Kleven et al. 2009). Briskie et al. (1997) hypothesized that longer sperm storage tubules enable female control over how sperm are used in fertilization. There is also a strong phylogenetic signal in the association between sperm length and sperm competition (Immler \& Birkhead 2007, Kleven et al. 2009, Lifjeld et al. 2010, Immler et al. 2012), which suggests that the role of sperm competition in sperm length evolution varies across the passerine phylogeny. 
More recently, studies have shown that increased levels of sperm competition are associated

with reduced inter- and intra-male variation in sperm length in passerine birds (Calhim et al. 2007, Immler et al. 2008, Kleven et al. 2008, Lifjeld et al. 2010), and also in insects (Fitzpatrick \& Baer 2011). Reduced variation in sperm length among males within a population suggests stronger stabilizing selection around an optimum length for high performance across different female environments (Calhim et al. 2007, Kleven et al. 2008, Lifjeld et al. 2010). In a comparative analysis, Lifjeld et al. (2010) showed that the coefficient of inter-male variation ( $\left.C V_{b m}\right)$ in sperm length explained as much as 65\% of the variation in extra-pair paternity rates among 24 passerine species. Since there was no phylogenetic signal in this association, Lifjeld et al. (2010) proposed that the $\mathrm{CV}_{\mathrm{bm}}$ metric could be used as a proxy for extra-pair paternity, and therefore sperm competition, in passerine birds. There is also a negative relationship between the coefficient of intra-male variation $\left(\mathrm{CV}_{\mathrm{wm}}\right)$ in sperm length and measures of sperm competition (Immler et al. 2008, Lifjeld et al. 2010). Reduced variation in sperm length within a male or an ejaculate should imply a stronger developmental stability or quality control in spermatogenesis.

In contrast to temperate species, we know surprisingly little about mating systems in tropical birds (Macedo et al. 2008). Stutchbury and Morton (2001) hypothesized that sperm competition levels should be generally lower in tropical than in temperate birds, but very few studies have actually tested this idea empirically (see Stutchbury et al. 1998, Stutchbury \& Morton 2001, Albrecht et al. 2013 for exceptions). Albrecht et al. (2013) found no difference in overall sperm competition levels between tropical and temperate passerine birds, using the sperm length $\mathrm{CV}_{\mathrm{bm}}$ index. They also noted that tropical species are apparently as variable as temperate zone birds in sperm competition levels, and mentioned specifically waxbills (Estrildidae) and sunbirds (Nectariniidae) as examples of families with low and high sperm competition levels, respectively. However, it is difficult to infer general patterns from just a few species; only three species of sunbirds were included in that study. General descriptive information about sperm morphology is also largely lacking for tropical birds. Moreover, 
tropical birds are relatively less well studied in terms of systematics (Reddy 2014) and general biology (Macedo et al. 2008).

Here, we examine variation in sperm morphology in 12 species of sunbirds from West Africa (Nigeria and Cameroon). Sunbirds are generally small (c. 5-22 g), socially monogamous species exhibiting a territorial breeding system (Fry et al. 2000, Cheke et al. 2001, Riegert et al. 2014). Additionally, the majority of species are sexually dimorphic in both body size and plumage coloration: males are larger and exhibit colourful iridescent plumage patches (either year round or seasonally), whereas females are generally drab (Fry et al. 2000, , Borrow \& Demey 2001, Cheke et al. 2001). The primary objectives of our study were to describe sperm length variation in sunbirds at multiple levels of organisation (i.e. among species and among and within males belonging to a single species) and test for signatures of phylogeny and sperm competition in the observed patterns of sperm morphological variation. We also tested for phenotypic correlates of sperm $\mathrm{CV}_{\mathrm{bm}}$ as a proxy for sperm competition.

\section{METHODS}

\section{Data collection and sampling procedure}

In Nigeria, fieldwork was conducted at Amurum Forest Reserve, Jos (09 53' N, $08^{\circ}$ 59' E); Yankari

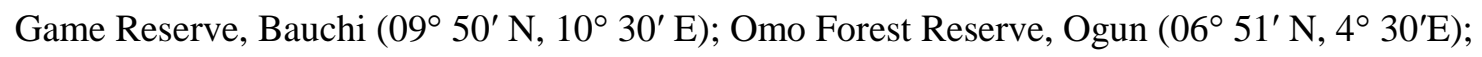

International Institute of Tropical Agriculture, Ibadan ( $\left.07^{\circ} 30^{\prime} \mathrm{N}, 03^{\circ} 55^{\prime} \mathrm{E}\right)$ and Okomu National Park, Benin $\left(06^{\circ} 33^{\prime} \mathrm{N}, 05^{\circ} 26^{\prime}\right)$. In Cameroon, we sampled birds along the slope of Mount Cameroon $\left(04^{\circ}\right.$ $\left.15^{\prime} \mathrm{N}, 09^{\circ} 09^{\prime} \mathrm{E}\right)$ and in the vicinity of Laide Farm, Bamenda-Banso Highlands ( $\left.06^{\circ} 05^{\prime} \mathrm{N}, 10^{\circ} 28^{\prime} \mathrm{E}\right)$. Birds were captured using mist-nets (in some instances with the assistance of song playback) during the breeding season (i.e. April to September in 2010-2013 in Nigeria and October to December in 2010-2012 in Cameroon). Sperm samples (c. 0.5-3 $\mu \mathrm{l}$ ) were collected by cloacal massage (Wolfson 1952) and immediately diluted in a small volume of phosphate-buffered saline (c. $20 \mu \mathrm{l})$ and then fixed in $300 \mu \mathrm{l}$ of $5 \%$ formaldehyde solution for later slide preparation. For each bird, a small blood sample (c. 10-50 $\mu$ l) was collected from the brachial vein and preserved in 96\% ethanol for later DNA 
extraction and DNA sequencing. We also fitted each bird with a uniquely numbered aluminium band (supplied by South African Bird Ringing Unit) to prevent resampling of individuals.

\section{Sperm morphology}

For each sample, a small aliquot (c. $15 \mu$ l) of formaldehyde-fixed sperm was applied to a glass slide and allowed to air-dry. Slides were then gently rinsed with distilled water and air-dried again. We captured high magnification (160× or 320×) digital images of sperm using a Leica DFC420 camera mounted on a Leica DM6000 B digital light microscope (Leica Microsystem, Heerbruug, Switzerland). We used Leica Application Suite (version 2.6.0 R1) to measure (to the nearest $\pm 0.1 \mu \mathrm{m}$ ) the length of the sperm head, midpiece and tail (i.e. the section of the flagellum not entwined by the midpiece), from which we calculated flagellum length (as the sum of midpiece and tail length), sperm total length (as sum of head, midpiece and tail length) and the ratios of flagellum:head length, midpiece:flagellum length and midpiece:total length.

For each male, we measured 10 morphologically normal spermatozoa following the recommendation in Laskemoen et al. (2007). All sperm measurements were taken by one person (TCO) to avoid observer effects. We determined the repeatability of sperm measurements by measuring the same 15 sperm from a single individual twice, and found that measurements were highly repeatable (head: $r=$ 0.87, $F_{14,15}=14.75, P<0.001$; midpiece: $r=0.81, F_{14,15}=9.76, P<0.001$, tail: $r=0.83, F_{14,15}=$ 10.94, $P<0.001$; Lessells \& Boag 1987). For each sperm trait we used the means within individuals to calculate the mean for each species. For two species we had sperm samples from both Nigeria and Cameroon. There were no significant differences between countries in sperm length or components for either species, but we used the Nigerian data only (larger $n$ ) for our comparative analyses. Finally, we calculated $\mathrm{CV}_{\mathrm{wm}}$ values of sperm total length for each individual and then used the mean of these values to calculate an average $\mathrm{CV}_{\mathrm{wm}}$ for each species. Similarly, we calculated the $\mathrm{CV}_{\mathrm{bm}}$ of sperm total length following the formula $\mathrm{CV}_{\mathrm{bm}}=(\mathrm{SD} /$ Mean $) * 100 *(1+(1 / 4 n))$, which corrects for variation in sample size (n) (Sokal \& Rohlf 1995). Phylogeny 
We sequenced the first part of the mitochondrial cytochrome oxidase I (COI) gene, which corresponds to the standard DNA barcode marker for animals (Hebert et al. 2003). Details of the DNA extraction, PCR and sequencing procedures are available as Appendix S1, Fig S1 and Table S1. To complement these data, but from different individuals, sequences were collected from another mitochondrial gene (NADH2) and three nuclear introns (FGB5, MB2, TGFb2) using standard protocols (Kimball et al. 2009, Fuchs et al. 2012). All COI sequences are publicly available at the BOLD database (Ratnasingham \& Hebert 2007) in the project folder BONSU. Data for the remaining loci are available on GenBank (xxxxxx-xxxxxx) Table S2. COI sequences were trimmed to an even length, and all loci were aligned using MAFFT v. 7 (Katoh \& Standley 2013), generating alignments for each locus of: COI: 654 base pairs, NADH2: 1041 bp, FGB5: 570 bp; MB2: 749 bp, TGFb2: 589 bp - Total 3603 bp); for 12 sunbird species and a flowerpecker (Flame-crowned Flowerpecker Dicaeum anthonyi), a member of the sister family to the sunbirds (Johansson et al. 2008) used to root the phylogenetic analyses described below. Species nomenclature follows the International Ornithologists’ Union (IOC) World Bird List (Gill \& Donsker 2015). A cross reference to names used by other checklists is presented in Table S3.

We estimated a maximum likelihood tree using the GTRGAMMA model of RaxML v. 8.1.24 (Stamatakis 2014) applied to the concatenated dataset using nine partitions (COI - codons 1, 2 \& 3; NADH2 - codons 1, 2 \& 3; FGB5, MB2, TGFb2). Analyses were conducted via the CIPRES Science Gateway supercomputer portal. To obtain a Bayesian tree and determine divergence times among species we made use of BEAST v 1.8.2 (Drummond et al. 2012) and the mean rates of divergence and associated standard deviations reported by Lerner et al. (2011) for each of the two mtDNA genes analysed and two of the introns (FGB and TGFb2). The rates reported by Lerner et al. (2011) are derived from the sequence of lineage splits in Hawaiian Honeycreepers (Fringillidae), and were calibrated using the well-established dates of sequential uplift of the Hawaiian Archipelago. The BEAST analyses was run for 100 million generations with a $\mathrm{HKY}+\mathrm{G}+\mathrm{I}$ model of nucleotide substitution applied to each locus, a strict molecular clock enforced and a Yule process for the tree 
prior. We made use of Tracer v.1.6.0 (Rambaut et al. 2014) to check that the effective sample size of the underlying posterior distribution was large enough (ESS > 200) for meaningful estimation of parameters.

\section{Sexual size dimorphism and plumage dichromatism}

We collected data on male and female body mass, wing length and sexual dichromatism from the literature (Fry et al. 2000, Cheke et al. 2001, Borrow \& Demey 2001, Cox et al. 2011). Sexual size dimorphism was estimated as the ratio of female body mass:male body mass and female wing length:male wing length, which we calculated using the mean values for each sex obtained from the literature. Next, we categorized plumage dichromatism as 0 or 1 , with 0 representing species that were monochromatic or showed only minor differences between the sexes (i.e. less than $10 \%$ of plumage differed) and 1 representing species that showed complete differences in colour or pattern (Table S4). Additionally, we scored male plumage ornamentation as the number of separate and distinct colour patches in the male plumage, i.e. head, throat-chest-belly and nape-back-rump. All plumage traits were assessed using image plates in Cheke et al. (2001). Finally, based on literature (Frey et al. 2000, Cheke et al. 2001), all species were assumed to be socially monogamous with the exception of the Olive Sunbird Cyanomitra olivacea and the Collared Sunbird Hedydipna collaris, which were classified as polygynous and polyandrous, respectively.

\section{Data analysis}

All analyses were performed using the statistical package R version 2.12.2 (R Development Core Team 2013). To improve data distributions, we log-transformed data prior to analysis. The ratios of midpiece:flagellum length, midpiece:total length, female:male body mass and female:male wing length were logit transformed following recommendation of Warton and Hui (2011). We tested for differences among species in sperm morphology (i.e. sperm total length and length of the various components) and $\mathrm{CV}_{\mathrm{wm}}$ using ANOVA. To assess whether species differed in $\mathrm{CV}_{\mathrm{bm}}$, we tested for 
homogeneity of variance in sperm length using Levene’s test. Next, for all sperm traits (i.e. head, midpiece, flagellum and total sperm length), we tested for the presence of a phylogenetic signal by calculating Blomberg's K (Blomberg et al. 2003), using the phylosig function in the 'phytools' package (Revell 2012): $K>1$ indicates that traits are more similar between related species than expected under Brownian motion evolution, whereas $K<1$ indicates high lability, at least at the tips of the tree (Blomberg et al. 2003). The presence of a phylogenetic signal was tested using a randomization test. We reconstructed the ancestral character state of sperm length using 'contMap' (Revell 2013). The mapping relies upon states estimated at internal nodes using maximum likelihood with 'fastAnc' and was plotted with 'contMap'.

The fit of five evolutionary models for the diversification of sperm length and sperm components in the time-calibrated phylogeny were compared against a null model of Brownian motion, using the fitContinuous function in the 'geiger' package (Harmon et al. 2008). These models were Lambda: phenotypic divergence covaries with phylogenetic distance, but allows for variable evolutionary rates; Delta: the evolutionary rate accelerates or decelerates over time; Kappa: evolutionary change occurs mainly at speciation events, and is not proportional to branch length; Ornstein-Uhlenbeck: a random walk within a constrained trait space, where traits tend to converge towards a single value; and Early Burst: an early burst of trait diversification followed by reduced evolutionary rates (or stasis). Models were compared using the Akaike Information Criterion corrected for small sample size (AICc); the model with the lowest AICc value indicates the best-fit model. We also calculated Akaike weights for all models and used both $\triangle$ AICc and Akaike weights values to assess model support. Values of $\Delta \mathrm{AICc} \leq 2$ are indicative of substantial support for the model (Burnham \& Anderson, 2004). For further details about the application of these models in another African passerine group, see Omotoriogun et al. (2016).

We performed phylogenetic generalized least-squares (PGLS) regressions using the package 'caper' (Orme et al. 2012), to examine the relationships among sperm traits and the relationships between sperm traits and $\mathrm{CV}_{\mathrm{bm}}$. For these latter models, separate models were run for each sperm trait. 
Similarly, we used PGLS regressions to determine whether measures of either sexual size dimorphism or sexual dichromatism predict sperm length $\mathrm{CV}_{\mathrm{bm}}$ (i.e. sperm competition) in sunbirds. This approach accounts for the statistical non-independence of data points due to shared ancestry of species (Pagel 1999, Freckleton et al. 2002). PGLS also allow for the estimation (via maximum likelihood) of the phylogenetic scaling parameter $\lambda$ ( $\lambda=0$ indicate phylogenetic independence, whereas $\lambda=1$ indicates phylogenetic dependence): superscript values in the reported results likelihood ratio tests for empirical $\lambda$ value against $\lambda=1$ and $\lambda=0$, respectively. Finally, we compared levels of $\mathrm{CV}_{\mathrm{bm}}$ in sunbirds to those of other passerine birds using a two-sample $t$-test. For this analysis, $\mathrm{CV}_{\mathrm{bm}}$ values for other species were extracted from Albrecht et al. (2013).

\section{RESULTS}

Sperm samples were analysed from a total of 189 males from 12 species belonging to five genera (Table 1, Table S5). Sperm total length ranged from $74 \mu \mathrm{m}$ in the Northern Double-collared Sunbird Cinnyris reichenowi to $116 \mu \mathrm{m}$ in the Scarlet-chested Sunbird Chalcomitra senegalensis, and differed significantly among species $\left(F_{11,177}=903.33, P<0.0001\right.$; Table 1$)$. The variation in sperm total length among species was largely explained by variation in midpiece and flagellum length, while head length was short in all species (range 12-14 $\mu$ m; Table 1, Fig. 1). However, all sperm components varied significantly among species $(P<0.0001$; Table 1$)$. Across the 12 species, head length was not correlated with midpiece length $\left(\beta=-0.02 \pm 0.02 \mathrm{SE}, t=-1.59, P=0.14, \lambda=0^{1.00 ;}\right.$; 0.12 ), flagellum length $\left(\beta=-0.03 \pm 0.02 \mathrm{SE}, t=-1.69, P=0.12, \lambda=0^{1.00 ; 0.01}\right)$ or sperm length $(\beta=-0.02 \pm$ $\left.0.02 \mathrm{SE}, t=-1.50, P=0.16, \lambda=0^{1.00 ; 0.11}\right)$. Furthermore, sperm total length was not associated with male body mass $\left(\beta=0.33 \pm 1.08 \mathrm{SE}, t=0.31, P=0.766, \lambda=1^{0.27 ; 1.00}\right)$. The tests are PGLS regressions controlling for the phylogeny depicted in Fig. 2.

Four species had sperm lengths $>100 \mu \mathrm{m}$ (Table 1), and since they all belong to different genera, there was no strong genus-specific differentiation in sperm lengths among our study species. However, when we mapped the sperm lengths onto the phylogeny derived from two mitochondrial 
genes and three nuclear introns (Fig. 2; see also the same phylogeny in Fig. S2 annotated with the 95\% highest probability density estimates for each node and rooted with the outgroup taxon), there was a trend that closely related species tended to have similar sperm lengths. Hence, there was also a significant phylogenetic signal in sperm length as estimated by Blomberg’s $K$ (Table 2). The phylogeny suggests that some sunbird genera are not monophyletic, e.g. the six Cinnyris species were spread across the entire phylogeny (Fig. 2), a result also supported with much greater taxon sampling (R.C.K. Bowie unpubl. data). Sperm midpiece and flagellum length, which are strongly intercorrelated with sperm total length, also showed a significant phylogenetic signal, but sperm head length did not (Table 2).

The tests of various evolutionary models supported a Brownian motion model of evolution for sperm total length and all components (all $\Delta \mathrm{AICc}=0.00$ and all $\Delta \mathrm{AICc}$ weights $>0.35$; Table 3 ). This implies that trait divergences did not consistently deviate from a random walk and was best predicted by the genetic distance between species or lineages.

The inter-male variance in sperm total length differed significantly among sunbird species (Levene's test: $F_{11,177}=2.518, P=0.006$ ). The $\mathrm{CV}_{\mathrm{bm}}$ in sperm length ranged from 1.49 to 3.50 for the nine species for which the metric was calculated (i.e. $n>3$; Table 1), with an average of $2.30 \pm 0.71$ $\mathrm{SD}$. The $\mathrm{CV}_{\mathrm{bm}}$ values for sunbirds did not differ significantly ( $t$-test: $t_{131}=-1.17, P=0.88$ ) from other passerine birds (i.e. 124 species in Albrecht et al. 2013, Table S1). There was no association between sperm $\mathrm{CV}_{\mathrm{bm}}$ and sexual size dimorphism (female:male body mass and wing length). Similarly, sperm $\mathrm{CV}_{\mathrm{bm}}$ was not associated with either sexual dichromatism or male plumage ornamentation (Table S6). Furthermore, sperm $\mathrm{CV}_{\mathrm{bm}}$ was not associated with sperm total length or any of its components, nor the flagellum:head ratio (Table 3). However, the sperm $\mathrm{CV}_{\mathrm{bm}}$ value was inversely correlated with relative midpiece length, and also a tendency in the same direction for absolute midpiece length (Fig. 3, Table 4). The intra-male variation $\left(\mathrm{CV}_{\mathrm{wm}}\right)$ in sperm length differed significantly among species and were generally quite low (range 1.19-2.50; Table 1$)$, but there was no correlation between intra-male $\left(\mathrm{CV}_{\mathrm{wm}}\right)$ 
and inter-male $\left(\mathrm{CV}_{\mathrm{bm}}\right)$ variation in sperm lengths across species $(\beta=0.23 \pm 0.17 \mathrm{SE}, t=1.32, P=0.23$, $\left.\lambda=0^{1.00 .00 ; 0.20}, n=9\right)$.

\section{DISCUSSION}

We have shown here how the length of sperm cells and their main structural components vary among and within 12 species of sunbird from West Africa. This is the first comparative analysis of sperm morphology from this family of birds (Nectariniidae), which encompasses altogether 143 species in Africa and the Oriental region (Gill \& Donsker 2015). Our results show significant variation in mean sperm total length among the species, within the range of 74-116 $\mu$ m (Table 1). Immler et al. (2011) listed sperm lengths for 196 passerine species in the range of 41.8-284.8 $\mu \mathrm{m}$. Sunbirds therefore have sperm length within the short-to-medium range for passerine birds.

We found evidence of a phylogenetic signal in the differentiation of sperm length among species (Table 2), which implies that species tend to differ more in sperm size the more distantly related they are in the phylogeny. There was also a significant phylogenetic signal in sperm midpiece and flagellum length, which constitute the larger parts of the sperm. We were not able to detect any significant deviation from a Brownian model of sperm evolution. This result stands in contrast to a recent study on sperm evolution in African greenbuls (Omotoriogun et al. 2016), that found evidence of lineage-specific rates of evolution in sperm length and generally more rapid differentiation around speciation events than along the branches in the phylogeny. It must be emphasized; however, that our sample of 12 sunbird species represents less than $10 \%$ of the total number of species in the family, so it is possible that a larger data set, with more statistical power and better resolution at the deeper nodes in the phylogeny, would detect other patterns of sperm evolution. At present, there is no clear theory for why the rate of sperm evolution should vary among groups of passerine birds.

Sperm heads were generally short and varied much less than other sperm components.

There was also no significant phylogenetic signal in sperm head length variation. There is a general trend among passerine birds that sperm head length is evolutionary conserved and varies within a 
rather narrow size range compared to the vast variation in midpiece and flagellum lengths (Jamieson 2006, Rowe et al. 2015). The head consists of the acrosome, which is functionally important in the fertilization process, and the nucleus, containing the haploid genome, which is normally densely packed (Jamieson 2006). Assuming drag is kept to the 'ideal' minimum level for swimming, the evolution of much longer flagella in some species could technically allow for an increase in head size, so there may be additional reasons for the conservation of short head lengths (Humphries et al. 2008). Our phylogeny suggests that some of the currently accepted taxonomic genera of sunbirds are not monophyletic. Recently, Lauron et al. (2015) noted the same pattern in a study of coevolution between malaria parasites and their sunbird hosts. Thus, there is clearly a need for more comprehensive studies of the sunbird phylogeny and an improved taxonomy.

Generally, passerine birds have higher rates of extra-pair paternity, i.e. more sperm competition, than other orders of birds, but the level of sperm competition is also variable among passerine species (Westneat \& Sherman 1997, Griffith et al. 2002). The sperm length $C V_{b m}$ metric carries information about the level of sperm competition (Calhim et al. 2007, Lifjeld et al. 2010), and it has recently been applied in several comparative analyses of sperm competition in passerine birds (Albrecht et al. 2013, Rowe et al. 2013, Gohli et al. 2013). Using the formula given in Lifjeld et al. (2010, Fig. 2), the minimum (1.49) and maximum (3.50) $\mathrm{CV}_{\mathrm{bm}}$-values observed for the sunbirds correspond to estimated frequencies of 39\% and 7\% extra-pair young, respectively, thus indicating a considerable span in the level of sperm competition. The average $\mathrm{CV}_{\mathrm{bm}}$-value of 2.30 calculated from nine sunbird species corresponds to a frequency of about 20\% extra-pair young, which is slightly higher than the average for passerine birds based on molecular paternity studies (Griffith et al. 2002). The three sunbird species reported in Albrecht et al. (2013) had a mean $\mathrm{CV}_{\mathrm{bm}}$ of 2.58 (range 2.26 2.76). Paternity studies from sunbirds are however limited. We are only aware of the study by Zilbermann et al. (1999) who found that 23\% of young in the Palestine Sunbird Cinnyris oseus were sired by extra-pair males, which makes a good match with our estimate. Extra-pair copulation behaviour is also reported from the Purple-rumped Sunbird Leptocoma zeylomica (Lamba 1978), and 
there are also observations of cloaca-pecking in sunbirds (Cheke et al. 2001), which may indicate multiple mating by females (cf. Davies 1984). $\mathrm{CV}_{\mathrm{bm}}$ values were lowest (and sperm competition levels presumably highest) for the two species that do not exhibit the typical socially monogamous mating system, i.e. the Olive Sunbird and the Collared Sunbird, which are considered socially polygynous and polyandrous, respectively (Cheke et al. 2001, Fry et al. 2000). Overall, it seems likely that sunbirds are characterized by mating systems where sperm competition is common, but that the level of sperm competition may vary with the social mating system. Sunbirds also tend to be sexually dimorphic in both size and plumage, but we found no significant associations between the $\mathrm{CV}_{\mathrm{bm}}$ index and measures of sexual size dimorphism or sexual dichromatism in our sample of species. phylogenetic relationships among species, we found one strong correlation with sperm competition that may suggest a role of selection. Relative midpiece size was greater in species with more sperm competition (i.e. lower $\mathrm{CV}_{\mathrm{bm}}$; Fig. 3). It is therefore possible that sperm competition favours the evolution of longer midpieces, with a higher mitochondrial loading of the sperm, which is important in sperm energetics (Rowe et al. 2013). Because the midpiece is wrapped around the flagellum, the flagellum needs to be as long as, or longer, than the midpiece for reasons of structural support. Selection for a longer midpiece will therefore as a consequence also imply selection for a longer flagellum, and hence a longer sperm. The correlation between sperm competition and relative midpiece size is therefore consistent with a trend among certain passerine groups, that sperm competition favours the evolution of longer sperm with a longer midpiece (Briskie et al. 1997, Kleven et al. 2009, Lifjeld et al. 2010, but see Immler \& Birkhead 2007). In conclusion, our study highlights a considerable inter-specific variation in mean sperm length and its variance across a sample of 12 sunbird species. The variation in sperm length reflects to a large extent the phylogenetic relationships among species. Differences in sperm length can therefore be explained by a neutral model of genetic drift, but there is also some indication that sperm competition drives the evolution of longer sperm through selection for a longer midpiece. We also 
found relatively low coefficients of inter-male variation in sperm length, which suggests that sperm competition is common in this group of birds.

This study was sponsored by the Research Council of Norway (project no. 196554/V40 to JTL), Norwegian State Educational Loan Fund (PhD scholarship to TCO), and the Czech Science Foundation (Project no. 14-36098G), International Foundation for Science (grant no. TJ/32343 to TCO) and the United States National Science Foundation (DEB-1120356 \& DEB-1441652 to RCKB). We thank the A.P. Leventis Ornithological Research Institute, University of Jos, for providing vehicle for our field work in Nigeria. The Nigeria National Park Service, Mt. Cameroon National Park authorities, and International Research and Training Centre, Yaoundé, Buea University granted permission to access field sites. Talatu Tende, Onoja Joseph, Taiye A. Adeyanju and Chima Nwaogu helped during our field work in Nigeria. Gunnhild Marthinsen, Silje Hogner and Lars Erik Johannessen assisted during laboratory analysis. We thank the Field Museum of Natural History and the Louisiana State University Museum of Natural Science, the University of Michigan Museum of Natural History, and Thomas Smith and Kevin Njabo for loan of tissue samples. Thanks to two anonymous referees and Rebecca Kimball for their helpful comments. This is contribution no XX from the A.P. Leventis Ornithological Research Institute.

\section{REFERENCES}

Albrecht, T., Kleven, O., Kreisinger, J., Laskemoen, T., Omotoriogun, T.C., Ottosson, U., Reif, J., Sedláček, O., Hořák, D., Robertson, R.J. \& Lifjeld, J.T. 2013. Sperm competition in tropical versus temperate zone birds. Proc. R. Soc. Lond. B 280: 20122434.

Balshine, S., Leach, B.J., Neat, F., Werner, N.Y. \& Montgomerie, R. 2001. Sperm size of African cichlids in relation to sperm competition. Behav. Ecol. 12: 726-731.

Blomberg, S.P., Garland, T. \& Ives, A.R. 2003. Testing for phylogenetic signal in comparative data: behavioral traits are more labile. Evolution 57: 717-745.

Borrow, N. \& Demey, R. 2001. The Birds of Western Africa. London, UK: Christopher Helm.

Briskie, J.V. \& Montgomerie, R. 1992. Sperm size and sperm competition in birds. Proc. R. Soc. Lond. B 247: 89-95.

Briskie , J.V., Montgomerie, R. \& Birkhead, T.R. 1997. The evolution of sperm size in birds. Evolution 51: 937-945. 
Calhim, S., Immler, S. \& Birkhead, T.R. 2007. Postcopulatory sexual selection is associated with reduced variation in sperm morphology. PLoS One 2: e413.

Cheke, R.A., Mann, C.F. \& Allen, R. 2001. A Guide to the Sunbirds, Flowerpeckers, Spiderhunters and Sugarbirds of the World, New Haven: Yale University Press.

Cohen, J. 1977. Reproduction. London: Butterworths.

Cox, D.T.C., Brandt, M.J., McGregor, R., Ottosson, U., Stevens, M.C. \& Cresswell, W. 2011. Patterns of seasonal and yearly mass variation in West African tropical savannah birds. Ibis 153: 672683.

Davies, N.B. 1984. Polyandry, cloaca-pecking and sperm competition in dunnocks. Nature 302: 334336.

Drummond, A.J., Suchard, M.A., Xie, D. \& Rambaut, A.J. 2012. Bayesian phylogenetics with BEAUti and BEAST 1.7. Mol. Biol. Evol. 29: 1969-1973.

Eberhard, W.G. 1996. Female Control: Sexual Selection by Cryptic Female Choice. Princeton, New Jersey: Princeton University Press.

Fitzpatrick, J.L. \& Baer, B. 2011. Polyandry reduces sperm length variation in social insects. Evolution 65: 3006-3012.

Franzén, Å. 1970. Phylogenetic aspects of the morphology of spermatozoa and spermiogenesis. In Baccetti, B. (ed.) Comparative Spermatology: 29-45. New York: Academic Press.

Freckleton, R.P., Harvey, P.H. \& Pagel, M. 2002. Phylogenetic analysis and comparative data: a test and review of evidence. Am. Nat. 160: 712-726.

Fry, C.H., Keith, S. \& Urban, E.K. 2000. The Birds of Africa. London: Academic Press.

Fuchs, J., Irestedt, M., Fjeldså, J., Couloux, A., Pasquet, E. \& Bowie, R.C.K. 2012. Molecular phylogeny of African bush-shrikes and allies: tracing the biogeographic history of an explosive radiation of corvoid birds. Mol. Phylogenet. Evol. 64: 93-105.

Gage, M.J.G. \& Freckleton, R.P. 2003. Relative testes size and sperm morphometry across mammals: no evidence for an association between sperm competition and sperm length. Proc. R. Soc. Lond. B 270: 625-632.

Gill, F. \& Donsker, D. 2015. IOC World Bird List (Version 5.3). Available at: www.worldbirdnames.org (accessed 27 September 2015).

Gohli, J., Anmarkrud, J.A., Johnsen, A., Kleven, O., Borge, T. \& Lifjeld, J.T. 2012. Female promiscuity is positively associated with neutral and selected genetic diversity in passerine birds. Evolution 67: 1406-1419.

Gomendio, M. \& Roldan, E.R.S. 1991. Sperm competition influences sperm size in mammals. Proc. $R$. Soc. Lond. B 243: 181-185.

Griffith, S.C., Owens, I.P.F. \& Thuman, K.A. 2002. Extra pair paternity in birds: a review of interspecific variation and adaptive function. Mol. Ecol. 11: 2195-2212.

Harmon, L.J., Weir, J.T., Brock, C.D., Glor, R.E. \& Challenger, W. 2008. GEIGER: investigating evolutionary radiations. Bioinformatics 24: 129-131.

Hebert, P., Ratnasingham, S. \& deWaard, J. 2003. Barcoding animal life: cytochrome c oxidase subunit 1 divergences among closely related species. Proc. R. Lond. B 270: S96-99.

Humphries, S., Evans, J.P. \& Simmons, L.W. 2008. Sperm competition: linking form to function. BMC Evol. Biol. 8: 319.

Immler, S. \& Birkhead, T. 2007. Sperm competition and sperm midpiece size: no consistent pattern in passerine birds. Proc. R. Soc. Lond. B 274: 561-568.

Immler, S., Calhim, S. \& Birkhead, T.R. 2008. Increased postcopulatory sexual selection reduces the intramale variation in sperm design. Evolution 62: 1538-1543.

Immler, S., Gonzalez-Voyer, A. \& Birkhead. T.R.2012. Distinct evolutionary patterns of morphometric sperm traits in passerine birds. Proc. R. Soc. Lond. B 279: 4174-4182. 
Immler, S., Pitnick, S., Parker, G.A., Durrant, K.L., Lüpold, S., Calhim, S. \& Birkhead, T.R. 2011. Resolving variation in the reproductive tradeoff between sperm size and number. Proc. Natl Acad. Sci. USA 108: 5325-5330.

Jamieson, B.G.M. 2006. Avian spermatozoa: structure and phylogeny. In Jamieson, B.G.M. (ed.) Reproductive Biology and Phylogeny of Aves: 249-511. Enfield, NH: Science Publishers Inc.

Johansson, U.S., Fjeldså, J. and Bowie, R.C.K. 2008. Phylogenetic relationships within Passerida (Aves: Passeriformes): a review and a new molecular phylogeny based on three nuclear intron markers. Mol. Phylogenet. Evol. 48: 858-876.

Katoh, K. \& Standley, D.M. 2013. MAFFT multiple sequence alignment software version 7: improvements in performance and usability. Mol. Biol. Evol. 30: 772-780.

Kimball, R.T., Braun, E.L., Barker, F.K., Bowie, R.C.K., Braun, M.J., Chojnowski, J.L., Hackett, S.J., Han, K-L., Harshman, J., Heimer-Torres, V., Holznagel, W., Huddleston, C.J. Marks, B.D., Miglia, K.J., Moore, W.S., Reddy, S., Sheldon, F.H., Smith, J.V., Witt, C.C. \& Yuri, T. 2009. A well-tested set of primers to amplify regions spread across the avian genome. Mol. Phylogenet. Evol. 50: 654-660.

Kleven, O., Fossøy, F., Laskemoen, T., Robertson, R.J., Rudolfsen, G. \& Lifjeld, J.T. 2009. Comparative evidence for the evolution of sperm swimming speed by sperm competition and female sperm storage duration in passerine birds. Evolution 63: 2466-2473.

Kleven, O., Laskemoen, T., Fossøy, F., Robertson, R.J. \& Lifjeld, J.T. 2008. Intraspecific variation in sperm length is negatively related to sperm competition in passerine birds. Evolution 62: 494-499.

Koehler, L.D. 1995. Diversity of avian spermatozoa ultrastructure with emphasis on the members of ther order Passeriformes. In Jamieson, B.G.M., Ausio, J. \& Justine, J.-L. (eds ) Advances in Spermatozoal Phylogeny and Taxonomy: 437-444. Paris: Muséum National d'Histoire Naturelle.

Lamba, B.S. 1978. Nidification of some common Indian birds 13: the Purple-rumped Sunbird, Nectarinia zeylonica Linnaeus. Indian J. For. 1: 329-344.

Laskemoen, T., Kleven, O., Foss $\varnothing$ y, F. \& Lifjeld, J.T. 2007. Intraspecific variation in sperm length in two passerine species, the Bluethroat Luscinia svecica and the Willow Warbler Phylloscopus trochilus. Orn. Fenn. 84: 131-139.

Lauron, E.J., Loiseau, C., Bowie, R.C.K., Spicer, G.S., Smith, T.B., Melo, M. \& Sehgal, R.N.M. 2015. Coevolutionary patterns and diversification of avian malaria parasites in African sunbirds (Family Nectariniidae). Parasitology 142: 635-647.

Lerner, H.R.L., Meyer, M., James, H. F., Hofreiter, M. \& Fleischer, R. C. 2011. Multilocus resolution of phylogeny and timescale in the extant adaptive radiation of Hawaiian honeycreepers. Curr. Biol. 21: 1838-1844.

Lessells, C.M. \& Boag, P.T. 1987. Unrepeatable repeatabilities: a common mistake. Auk 104: 116121.

Lifjeld, J.T., Laskemoen, T., Kleven, O., Albrecht, T. \& Robertson, R.J. 2010. Sperm length variation as a predictor of extrapair paternity in passerine birds. PLOS ONE 5: e13456.

Lüpold, S., Calhim, S., Immler, S. \& Birkhead, T.R. 2009. Sperm morphology and sperm velocity in passerine birds. Proc. R. Soc. Lond. B 276: 1175-1181.

Lüpold, S., Manier, M.K., Berben, K.S., Smith, K.J., Daley, B.D., Buckley, S.H., Belote, J.M. \& Pitnick, S. 2012. How multivariate ejaculate traits determine competitive fertilization success in Drosophila melanogaster. Curr. Biol. 22: 1667-1672.

Miller, G.T. \& Pitnick, S. 2002. Sperm-female coevolution in Drosophila. Science 298: 1230-1233.

Macedo, R.H., Karubian, J. \& Webster, M.S. 2008. Extrapair paternity and sexual selection in socially monogamous birds: are tropical birds different? Auk 125: 769-777. 
Morrow, E.H. \& Gage, M.J.G. 2000. The evolution of sperm length in moths. Proc. R. Soc. Lond. B 267: 307-313.

Omotoriogun, T.C., Albrecht, T., Hořák, D., Laskemoen, T., Ottosson, U., Rowe, M., Sedláček, O. \& Lifjeld, J.T. 2016. Sperm size evolution in African greenbuls (Passeriformes: Pycnonotidae). Biol. J. Linn. Soc. xx: xxx-xxx. (DOl: 10.1111/bij.12663)

Orme, C.D.L., Freckleton, R.P., Thomas, G.H., Petzoldt, T., Fritz, S.A., Isaac, N.J.B. \& Pearse, W. 2012 caper: comparative analyses of phylogenetics and evolution in R. Methods Ecol. Evol. 3: 145151.

Pagel, M. 1999. Inferring the historical patterns of biological evolution. Nature 401: 877-884.

Parker, G.A. 1970. Sperm competition and its evolutionary consequenses in the insects. Biol. Rev. 45: 525-567.

Parker, G.A. 1993. Sperm competition games: sperm size and number under adult control. Proc. $R$. Soc. Lond. B 253: 245-254.

Parker, G.A. 1998. Sperm competititon and the evolution of ejaculates: towards a theory based. In Birkhead, T.R. \& Møller, A.P. (eds) Sperm Competition and Sexual Selection: 3-54. Academic Press.

Pitnick, S., Hosken, D.J. \& Birkhead, T.R. 2009. Sperm morphological diversity. In Birkhead, T.R., Hosken, D.J. \& Pitnick, S. (eds) Sperm Biology, an Evolutionary Perspective: 69-149. San Diego, CA: Academic Press.

Pizzari, T. \& Parker, G.A. 2009. Sperm competition and sperm phenotype. In Birkhead, T.R., Hosken, D.J. \& Pitnick, S. (eds) Sperm Biology, an Evolutionary Perspective: 205-245. London: Elsevier.

Ratnasingham, S. \& Hebert, P. D. N. 2007. BOLD: The Barcode of Life Data System (www.barcodinglife.org). Molecular Ecology Notes 7: 355-364.

R Development Core Team. 2013. R: A Language and Environment for Statistical computing, Vienna: R Foundation for Statistical Computing. Available at: www.R-project.org.Rambaut, A., Suchard, M.A., Xie, D. \& Drummond, A.J. 2014. Tracer v1.6, Available at: http://beast.bio.ed.ac.uk/Tracer.

Rambaut, A. \& Drummond, A.J. 2009. "TreeAnnotator v1. 6.2: MCMC output analysis.

Reddy, S. 2014. What's missing from avian global diversification analyses? Mol. Phyl. Evol. 77: 159165.

Revell, L.J. 2012. Phytools: phylogenetic tools for comparative biology (and other things). Methods Ecol. Evol. 3: 217-223.

Revell, L. J. 2013. Two new graphical methods for mapping trait evolution on phylogenies. Methods Ecol. Evol. 4: 754-759.

Riegert, J., Antczak, M., Fainová, D. \& Blažková, P. 2014. Group display in the socially monogamous Northern Double-collared Sunbird Cinnyris reichenowi. Behavioural Processes 103: 138-144.

Rowe, M., Albrecht, T., Cramer, E.R.A., Johnsen, A., Laskemoen, T., Weir, J.T. \& Lifjeld, J.T. 2015. Postcopulatory sexual selection is associated with accelerated evolution of sperm morphology. Evolution 69: 1044-1052.

Rowe, M., Laskemoen, T., Johnsen, A. \& Lifjeld, J.T. 2013. Evolution of sperm structure and energetics in passerine birds. Proc. R. Soc. Lond. B 280 (1753): 20122616.

Snook, R.R. 2005. Sperm in competition: not playing by the numbers. Trends Ecol. Evol. 20: 46-53.

Sokal, R.R. \& Rohlf, F.J. 1995. Biometry: the Principles and Practice of Statistics in Biological Research. New York, NY: Freeman.

Stamatakis A. 2014 RAxML version 8: a tool for phylogenetic analysis and post-analysis of large phylogenies. Bioinformatics. 30: 1312-1313.

Stutchbury, B.J.M. \& Morton, E.S. 2001. Behavioural Ecology of Tropical Birds. San Diego, California: Academic Press. 
Stutchbury, B.J.M., Morton, E.S. \& Piper, W.H. 1998. Extra-pair mating system of a synchronously breeding tropical songbird. J. Avian Biol. 29: 72-78.

Stutchbury, B. \& Morton, E. 2008. Recent advances in the behavioral ecology of tropical birds. The Wilson Journal of Ornithology 120: 26-37.

Tourmente, M., Gomendio, M. \& Roldan, E. 2011. Sperm competition and the evolution of sperm design in mammals. BMC Evolutionary Biology 11: 12.

Warton, D.I. \& Hui, F.K.C. 2011. The arcsine is asinine: the analysis of proportions in ecology. Ecology 92: 3-10.

Westneat, D.F. \& Sherman, P.W. 1997. Density and extra-pair fertilizations in birds: a comparative analysis. Behav. Evol. Sociobiol. 41: 205-215.

Wolfson, A. 1952. The cloacal protuberance - a means for determining breeding condition in live male passerines. Bird-Banding 23: 159-165.

Zilberman, R., Moav, B. \& Yom-Tov, Y. 1999. Extra-pair paternity in the socially monogamous Orange-tufted Sunbirds Nectarinia osea osea. Israel Journal of Zoology 45: 407-421. 


\section{SUPPORTING INFORMATION}

Additional Supporting Information may be found in the online version of this article:

Appendix S1. Detail of DNA extraction, PCR, sequencing the mitochondrial COI gene and phylogeny construction of sunbirds.

Fig. S1. A maximum likelihood tree of 15 species of sunbirds based on the mitochondrial COI gene.

Fig. S2. A Bayesian tree based on the concatenated sequences from two mitochondrial genes (COI and NADH2) and three nuclear introns (FGB5, MB2, TGFb2) and with 95\% highest probability densities (HPD) estimated around each mean divergence time for each node.

Table S1. Voucher information of the samples used for sequencing the mitochondrial COI gene.

Table S2. Voucher information of the samples used for sequencing the mitochondrial NADH2 gene and three nuclear introns (FGB5, MB2, TGFb2).

Table S3. The common and species name of sunbirds according to the IOC World Bird List, and with cross reference to Taxonomy in Flux, BirdLife International and Internet Bird Collection checklists.

Table S4. Plumage categories used in the analysis testing for association between inter-male coefficient of variation of sperm length $\left(\mathrm{CV}_{\mathrm{bm}}\right)$ and plumage dichromatism in sunbirds ( $\mathrm{N}=9$ species). Sexual dichromatism scored as monochromatic (0) or dichromatic (1). Male plumage ornamentation scored as the number of distinct colour patches on the male plumage. Scores were based on plate illustrations of adult birds in Cheke et al. (2001).

Table S5. Detail of individual male sperm morphology data analysed for 12 species of sunbird. Length $(\mu \mathrm{m})$ of sperm head, midpiece, flagellum and total are based on the average of 10 spermatozoa measured per individual. The $\mathrm{CV}_{\mathrm{wm}}$ is intra-male coefficient of variation of sperm total length.

Table S6. Regression analysis controlling for phylogeny (PGLS) between inter-male coefficient of variation of sperm length and sexual size dimorphism, and sexual dichromatism in sunbirds ( $\mathrm{N}=9$ species). The model including the maximum-likelihood of lambda $(\lambda)$ value was compared against the models including $\lambda=1$ and $\lambda=0$, and superscripts following the $\lambda$ values indicate probability $(P)$ of likelihood-ratio of indices of sexual size dimorphism or plumage dichromatism (first position: against $\lambda=0$; second position: against $\lambda=1$ ). 
Table 1. Descriptive statistics (mean \pm SD) of sperm traits for 12 species of sunbird with tests of species differences (ANOVA). Lengths are given in $\mu$ m, coefficients of intra-male $\left(\mathrm{CV}_{\mathrm{wm}}\right)$ and inter-male $\left(\mathrm{CV}_{\mathrm{bm}}\right)$ variation in sperm total length are given in percent.

\begin{tabular}{|c|c|c|c|c|c|c|c|}
\hline Species & Country & Head length & Midpiece length & Flagellum length & Total length & $\mathrm{CV}_{\text {wm }}$ (total length) & $\mathrm{CV}_{\mathrm{bm}}$ (total length) \\
\hline Chalcomitra senegalensis $(n=66)$ & Nigeria & $12.83 \pm 0.55$ & $86.07 \pm 3.10$ & $102.97 \pm 2.37$ & $115.62 \pm 2.83$ & $1.61 \pm 0.47$ & 2.03 \\
\hline Cinnyris bouvieri $(n=7)$ & Cameroon & $12.05 \pm 0.47$ & $65.06 \pm 1.62$ & $79.00 \pm 1.86$ & $91.05 \pm 1.88$ & $2.01 \pm 0.55$ & 2.13 \\
\hline Cinnyris coccinigastrus $(n=1)$ & Nigeria & 12.21 & 79.52 & 91.09 & 103.30 & 1.55 & \\
\hline Cinnyris cupreus $(n=7)$ & Nigeria & $12.08 \pm 0.25$ & $57.90 \pm 2.21$ & $74.61 \pm 1.64$ & $86.69 \pm 1.75$ & $1.65 \pm 0.30$ & 2.09 \\
\hline Cinnyris reichenowi $(n=16)$ & Cameroon & $12.42 \pm 0.48$ & $47.78 \pm 2.14$ & $61.69 \pm 1.59$ & $74.11 \pm 1.71$ & $2.32 \pm 1.38$ & 2.35 \\
\hline Cinnyris ursulae $(n=1)$ & Cameroon & 13.44 & 54.74 & 70.81 & 84.26 & 1.19 & \\
\hline Cinnyris venustus $(n=4)$ & Nigeria & $12.73 \pm 0.26$ & $67.57 \pm 2.68$ & $82.11 \pm 1.70$ & $94.84 \pm 1.82$ & $2.17 \pm 1.07$ & 2.04 \\
\hline Cyanomitra olivacea $(n=49)$ & Nigeria & $13.80 \pm 0.47$ & $60.46 \pm 1.42$ & $72.28 \pm 1.37$ & $86.08 \pm 1.27$ & $1.54 \pm 0.42$ & 1.49 \\
\hline${ }^{\dagger}$ Cyanomitra olivacea $(n=16)$ & Cameroon & $13.80 \pm 0.42$ & $60.37 \pm 1.51$ & $72.61 \pm 1.53$ & $86.41 \pm 1.54$ & $1.49 \pm 0.30$ & 1.78 \\
\hline Cyanomitra oritis $(n=18)$ & Cameroon & $12.91 \pm 0.63$ & $48.74 \pm 2.82$ & $68.75 \pm 2.59$ & $81.66 \pm 1.91$ & $2.50 \pm 1.75$ & 3.50 \\
\hline Cyanomitra verticalis $(n=9)$ & Nigeria & $12.59 \pm 1.08$ & $54.37 \pm 2.67$ & $71.12 \pm 2.91$ & $83.62 \pm 2.77$ & $1.62 \pm 0.59$ & 3.41 \\
\hline Deleornis fraseri $(n=3)$ & Nigeria & $11.50 \pm 0.47$ & $79.18 \pm 1.25$ & $96.80 \pm 2.36$ & $108.30 \pm 2.72$ & $1.62 \pm 0.63$ & \\
\hline${ }^{\dagger}$ Deleornis fraseri $(n=2)$ & Cameroon & $12.47 \pm 0.58$ & $83.54 \pm 3.24$ & $100.32 \pm 3.30$ & $112.78 \pm 2.72$ & $1.68 \pm 0.22$ & \\
\hline \multirow[t]{2}{*}{ Hedydipna collaris $(n=8)$} & Nigeria & $11.35 \pm 0.55$ & $82.39 \pm 1.39$ & $96.37 \pm 1.63$ & $107.71 \pm 1.69$ & $1.69 \pm 0.19$ & 1.62 \\
\hline & & $F_{11,177}=23.13$ & $F_{11,177}=741.16$ & $F_{11,177}=959.18$ & $F_{11,177}=903.33$ & $F_{11,177}=2.92$ & \\
\hline ANOVA & & $P<0.0001$ & $P<0.0001$ & $P<0.0001$ & $P<0.0001$ & $P=0.002$ & \\
\hline
\end{tabular}

${ }^{\dagger}$ Measurements of sperm traits for these populations were not included in the ANOVA test and comparative (PGLS) analysis. 
Table 2. Test of phylogenetic signal in sperm traits among sunbirds ( $n=12$ species) using Blomberg's $K$ ( $P$-values for randomization test).

\begin{tabular}{|c|c|c|}
\hline \multirow[b]{2}{*}{ Sperm traits } & \multicolumn{2}{|c|}{ Blomberg's $K$} \\
\hline & $K$ & $P$ (Randomization) \\
\hline Head length & 0.881 & 0.762 \\
\hline Midpiece length & 1.263 & 0.012 \\
\hline Flagellum length & 1.238 & 0.019 \\
\hline Total length & 1.231 & 0.013 \\
\hline
\end{tabular}


Table 3. Tests of various evolutionary models for sperm length diversification in 12 species of sunbirds using the fitContinuous function in the 'geiger' package (Harmon et al. 2008). For each sperm trait, the model with the lowest AICc value (i.e., $\triangle \mathrm{AICc}=0$ ) is considered the best-fitting model (boldface with *). The parameters estimated by the models are: $\sigma^{2}=$ net rate of trait evolution in Brownian motion model or the initial rate of evolution in the Early Burst model, $\lambda=$ extent to which the phylogeny predicts covariance among traits for species, $\delta=$ compares the contribution of early versus late trait evolution across a phylogeny, $\kappa=$ evolutionary change in trait associated with speciation events along the branch-length in the Kappa models, $\alpha=$ evolutionary constraint parameter in the Ornstein-Uhlenbeck model moving trait values back to the optimum and $\mathrm{r}=$ change in rate of trait evolution through time in the Early Burst model.

\begin{tabular}{|c|c|c|c|c|c|}
\hline \multirow[b]{2}{*}{ Models } & \multirow[b]{2}{*}{ Parameters } & \multicolumn{4}{|c|}{ Length of sperm traits } \\
\hline & & Head & Midpiece & Flagellum & Total sperm \\
\hline \multirow{3}{*}{$\begin{array}{l}\text { Brownian } \\
\text { motion }\end{array}$} & $\triangle \mathrm{AICc}$ & 0.000* & 0.000* & 0.000* & 0.000* \\
\hline & AICc weight & 0.461 & 0.494 & 0.529 & 0.545 \\
\hline & $\sigma^{2}$ & 0.0001 & 0.0053 & 0.0033 & 0.0024 \\
\hline \multirow[t]{4}{*}{ Lambda } & $\triangle \mathrm{AICc}$ & 2.703 & 3.929 & 3.929 & 3.929 \\
\hline & AICc weight & 0.119 & 0.0692 & 0.0742 & 0.0764 \\
\hline & $\lambda$ & $<0.0001$ & 1.0000 & 1.0000 & 1.0000 \\
\hline & $\sigma^{2}$ & 0.0004 & 0.0053 & 0.0033 & 0.0024 \\
\hline \multirow[t]{4}{*}{ Delta } & $\triangle \mathrm{AICc}$ & 2.772 & 3.616 & 3.532 & 3.585 \\
\hline & AICc weight & 0.115 & 0.0809 & 0.0905 & 0.0908 \\
\hline & $\delta$ & 2.99 & 0.5103 & 0.4657 & 0.4873 \\
\hline & $\sigma^{2}$ & 0.0004 & 0.0072 & 0.0047 & 0.0033 \\
\hline \multirow[t]{4}{*}{ Карра } & $\triangle \mathrm{AICc}$ & 3.9286 & 3.9286 & 3.9286 & 3.9286 \\
\hline & AICc weight & 0.1195 & 0.0692 & 0.0742 & 0.0765 \\
\hline & $\kappa$ & 1.0000 & 1.0000 & 1.0000 & 1.0000 \\
\hline & $\sigma^{2}$ & 0.0006 & 0.0053 & 0.0033 & 0.0024 \\
\hline \multirow{4}{*}{$\begin{array}{l}\text { Ornstein- } \\
\text { Uhlenbeck }\end{array}$} & $\triangle \mathrm{AICc}$ & 2.703 & 3.929 & 3.929 & 3.929 \\
\hline & AICc weight & 0.1195 & 0.06924 & 0.0742 & 0.0765 \\
\hline & $\alpha$ & 20.978 & $<0.0001$ & $<0.0001$ & $<0.0001$ \\
\hline & $\sigma^{2}$ & 0.1275 & 0.00530 & 0.00335 & 0.00239 \\
\hline \multirow[t]{4}{*}{ Early Burst } & $\triangle \mathrm{AICc}$ & 3.9285 & 1.63741 & 2.423338 & 2.798855 \\
\hline & AICc weight & 0.0647 & 0.2177 & 0.1575 & 0.1345 \\
\hline & $\mathrm{r}$ & 0.00 & -0.6980 & -0.50322 & -0.42942 \\
\hline & $\sigma^{2}$ & 0.00057 & 0.07819 & 0.025182 & 0.01401 \\
\hline
\end{tabular}


Table 4. Regression analysis controlling for phylogeny (PGLS) between the sperm length $\mathrm{CV}_{\mathrm{bm}}$ index (predictor) and sperm size traits in sunbirds ( $n=9$ species). The model including the maximum-likelihood values or lambda $(\lambda)$ value was compared against $\lambda=1$ and $\lambda=0$, with superscripts following the $\lambda$ values indicating the probability $(P)$ of likelihood-ratio of sperm trait (first position: against $\lambda=0$; second position: against $\lambda=1$ ).

\begin{tabular}{lccll}
\hline Sperm traits & \multicolumn{1}{l}{$\beta \pm \mathrm{SE}$} & \multicolumn{1}{l}{$P$} & \multicolumn{1}{l}{$0^{1.00 ; 0.19}$} \\
\hline Head & $0.05 \pm 0.36$ & 0.14 & 0.89 & $0^{1.00 ; 0.51}$ \\
Midpiece & $-11.36 \pm 5.89$ & -1.92 & 0.09 & $0^{1.00 ; 0.60}$ \\
Flagellum & $-8.64 \pm 6.32$ & -1.37 & 0.21 & $0^{1.00 ; 0.50}$ \\
Total length & $-8.59 \pm 6.22$ & -1.38 & 0.21 & $0^{1.00 ; 0.47}$ \\
Flagellum:head & $-0.75 \pm 0.59$ & -1.27 & 0.24 & $0^{1.00 ; 0.24}$ \\
Midpiece:flagellum & $-0.34 \pm 0.08$ & -4.58 & 0.003 & $0^{1.00 ; 0.35}$ \\
Midpiece:total length & $-0.28 \pm 0.08$ & -3.33 & 0.013 & \\
\hline
\end{tabular}




\section{FIGURE LEGENDS}

Figure 1. Relationship between sperm total length and sperm head, midpiece and flagellum length among sunbirds ( $n=12$ species). Data points represent species means.

Figure 2. Reconstruction of sperm length in colour gradients along branches and nodes of the phylogeny of 12 sunbird species. The legend shows the colour range from red (short sperm) to blue (long sperm). The length of the legend also provides a scale for the branch-lengths in million years (mya). The phylogeny is based on a Bayesian tree constructed from five concatenated genes and rooted with the Flame-crowned Flowerpecker Dicaeum anthonyi (for details see Methods); the mean divergence times with 95\% highest probability density estimates are available in the Supplementary information (Fig. S2).

Figure 3. The relationship between the coefficient of inter-male variation of sperm total length $\left(\mathrm{CV}_{\mathrm{bm}}\right)$ and (a) midpiece length, and (b) sperm midpiece:flagellum ratio in sunbirds ( $n=9$ species). Data points represent species means. For statistical tests, see Table 4. 


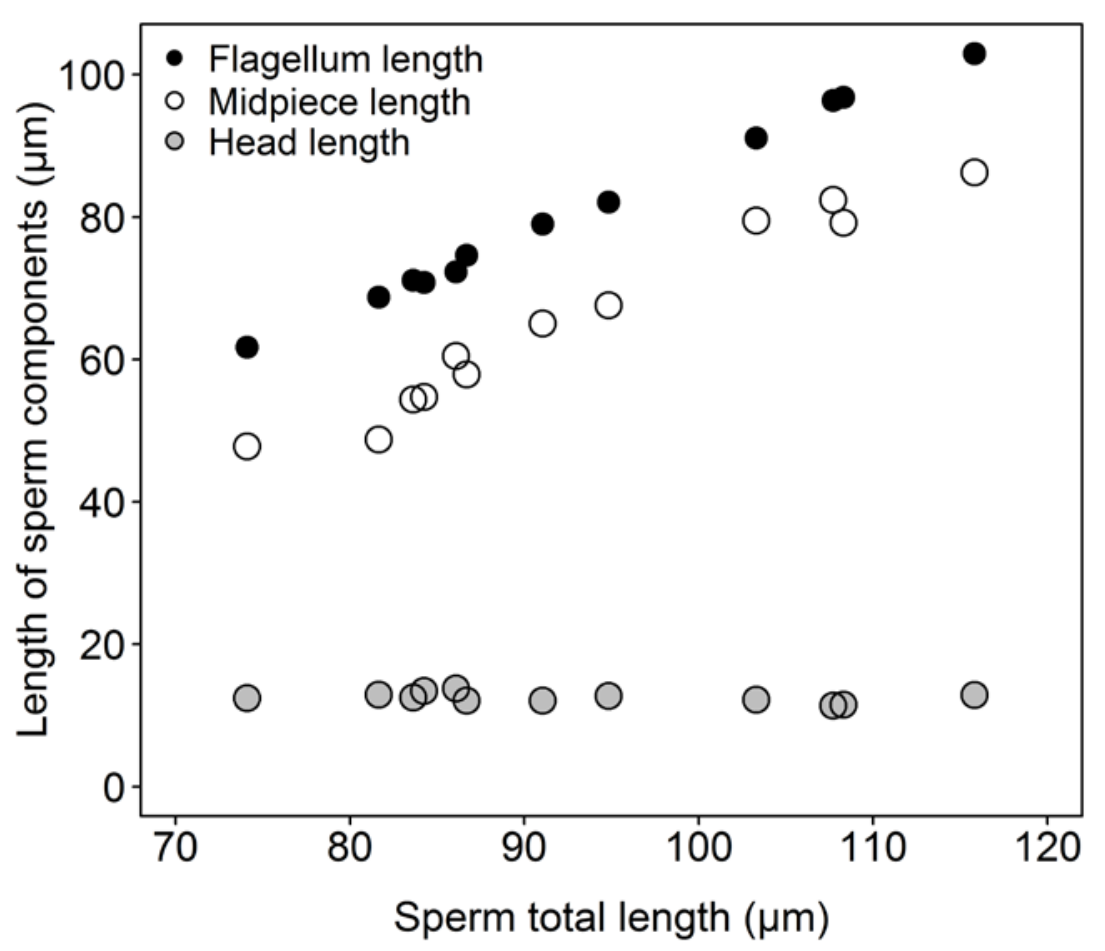

Figure 1 


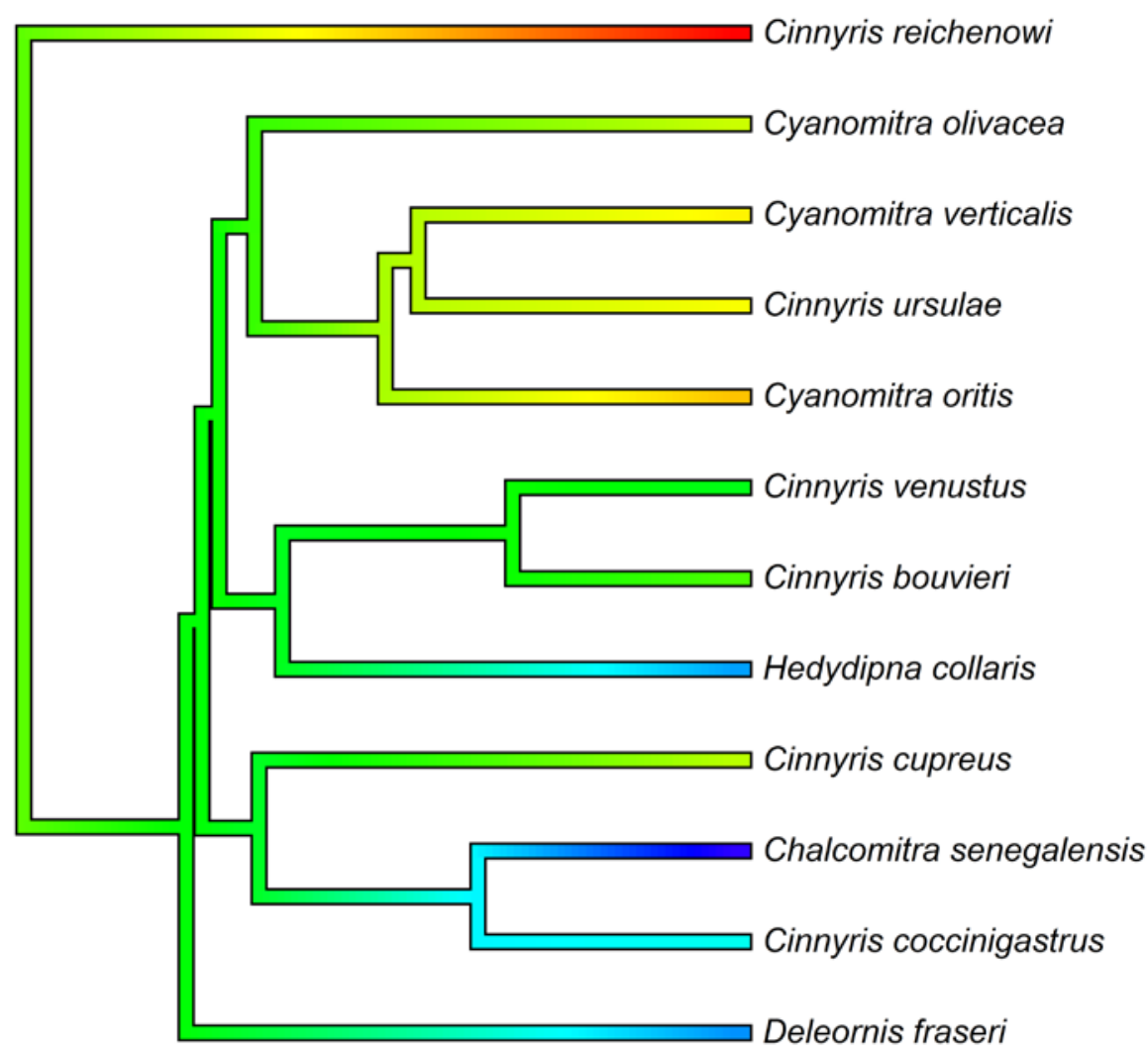

74.11 sperm total length $(\mu \mathrm{m}) 115.81$

length $=3$ mya

Figure 2 

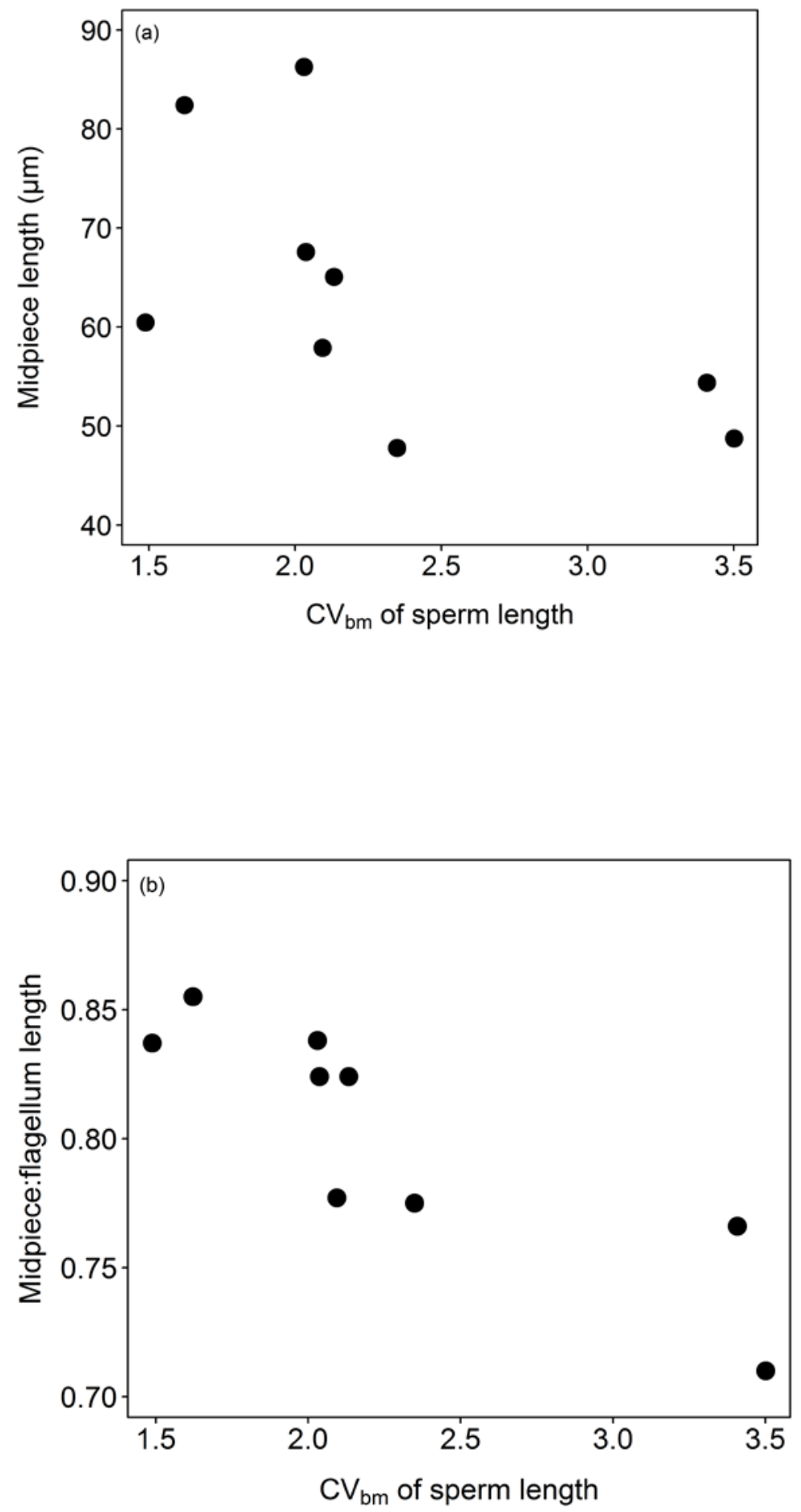

Figure 3 